\title{
Clustering and Symbolic Analysis of Cardiovascular Signals: Discovery and Visualization of Medically Relevant Patterns in Long-Term Data Using Limited Prior Knowledge
}

\author{
Zeeshan Syed, ${ }^{1}$ John Guttag, ${ }^{1}$ and Collin Stultz ${ }^{1,2}$ \\ ${ }^{1}$ Massachusetts Institute of Technology, Cambridge, MA 02139-4307, USA \\ ${ }^{2}$ Brigham and Women's Hospital, Cambridge, MA 02115, USA
}

Received 30 April 2006; Revised 18 December 2006; Accepted 27 December 2006

Recommended by Maurice Cohen

This paper describes novel fully automated techniques for analyzing large amounts of cardiovascular data. In contrast to traditional medical expert systems our techniques incorporate no a priori knowledge about disease states. This facilitates the discovery of unexpected events. We start by transforming continuous waveform signals into symbolic strings derived directly from the data. Morphological features are used to partition heart beats into clusters by maximizing the dynamic time-warped sequence-aligned separation of clusters. Each cluster is assigned a symbol, and the original signal is replaced by the corresponding sequence of symbols. The symbolization process allows us to shift from the analysis of raw signals to the analysis of sequences of symbols. This discrete representation reduces the amount of data by several orders of magnitude, making the search space for discovering interesting activity more manageable. We describe techniques that operate in this symbolic domain to discover rhythms, transient patterns, abnormal changes in entropy, and clinically significant relationships among multiple streams of physiological data. We tested our techniques on cardiologist-annotated ECG data from forty-eight patients. Our process for labeling heart beats produced results that were consistent with the cardiologist supplied labels $98.6 \%$ of the time, and often provided relevant finer-grained distinctions. Our higher level analysis techniques proved effective at identifying clinically relevant activity not only from symbolized ECG streams, but also from multimodal data obtained by symbolizing ECG and other physiological data streams. Using no prior knowledge, our analysis techniques uncovered examples of ventricular bigeminy and trigeminy, ectopic atrial rhythms with aberrant ventricular conduction, paroxysmal atrial tachyarrhythmias, atrial fibrillation, and pulsus paradoxus.

Copyright (c) 2007 Zeeshan Syed et al. This is an open access article distributed under the Creative Commons Attribution License, which permits unrestricted use, distribution, and reproduction in any medium, provided the original work is properly cited.

\section{INTRODUCTION}

The increasing prevalence of long-term monitoring in both ICU and ambulatory settings will yield ever increasing amounts of physiological data. The sheer volume of information that is generated about an individual patient poses a serious challenge to healthcare professionals. Patients in an ICU setting, for example, often have continuous streams of data arising from telemetry monitors, pulse oximeters, SwanGanz catheters, and arterial blood gas lines-to name just a few sources.

Any process that requires humans to examine more than small amounts of data is highly error prone. It is therefore not surprising that errors have been associated with "information overload" and that clinically relevant events are often missed $[1,2]$. Computer-based systems can be used to detect some events, but most conventional algorithms are tailored to detect specific classes of disorders.

In this paper, we describe a new approach to analyzing large sets consisting of physiological data relating to the cardiovascular system. We rely on morphologic characteristics of the physiological signal. However, unlike traditional expert systems, which can be used to search for a prespecified set of events using a priori knowledge, our approach allows for the discovery of events that do not need to be specified in advance. Our interest in techniques that do not incorporate knowledge about the events to be detected is motivated by a desire to uncover physiological activity that may have potential impact on patient care, but would not be detected by conventional methods.

The techniques that we present can be used to discover interesting events over long periods of time. We focus 
(a)

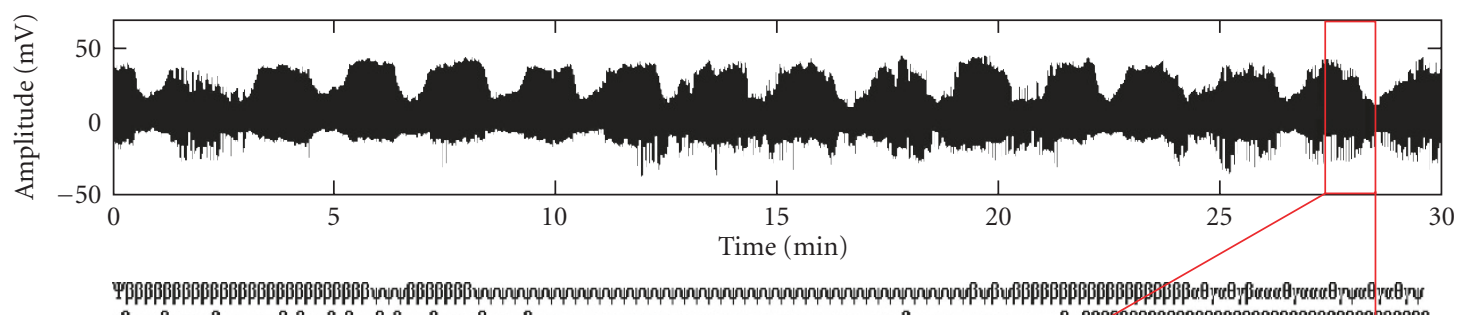

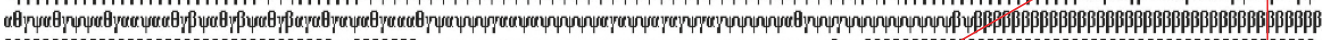

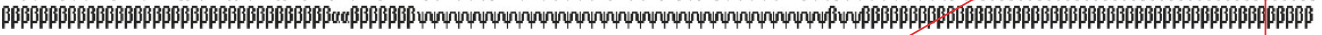

(b)

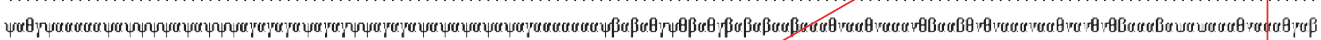
uө

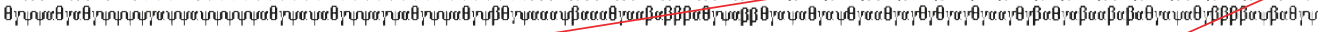

(c) $\theta \gamma \beta \alpha \theta \gamma \beta \alpha \theta \gamma \beta \beta \theta \gamma \beta \alpha \theta \gamma \beta \alpha \theta \gamma \alpha \theta \gamma \beta \alpha \theta \gamma \beta \theta \gamma \beta \alpha \theta \gamma \beta \beta \theta \gamma \beta \alpha \theta \gamma \beta \beta \theta \gamma \beta \alpha \theta \gamma \beta \alpha$

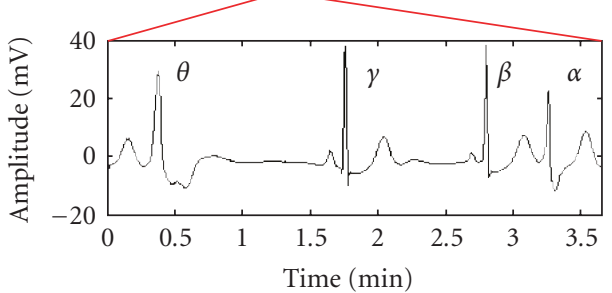

(d)

FIGURE 1: Overview of symbolic analysis: (a) raw data corresponding to Patient 106 in the MIT-BIH arrhythmia database. The red rectangle denotes a particular pattern hidden within the raw data. This pattern is difficult to identify by visual examination alone. (b) The raw ECG data is mapped into a symbolic representation (11 lines of the symbol sequence are elided from this figure). (c) An example rhythm of a repeating sequence, found in the symbolized representation of the data corresponding to the boxed area of the raw data in (a). (d) An archetypal representation, created using the techniques in [3], of the repeating signal.

primarily on the analysis of ECG data, extending our work to other signals in multiparameter datasets to find cross-signal interactions.

We propose a two-step process for discovering relevant information in cardiovascular datasets. As a preliminary step, we segment physiological signals into basic quasiperiodic units (e.g., heart beats recorded on ECG). These units are partitioned into classes using morphological features. This allows the original signal to be reexpressed as a symbolic string, corresponding to the sequence of labels assigned to the underlying units.

The second step involves searching for significant patterns in the reduced representation resulting from symbolization. In the absence of prior knowledge, significance is assessed by organization of basic units as adjacent repeats, frequently occurring words, or subsequences that cooccur with activity in other signals. The fundamental idea is to search for variations that are unlikely to occur purely by chance as such patterns are most likely to be clinically relevant. The abstraction of cardiovascular data as a symbolic string allows efficient algorithms from computational biology and information theory to be leveraged.

Figure 1 presents an overview of this approach. We start by using conventional techniques to segment an ECG signal into individual beats. The beats are then automatically partitioned into classes based upon their morphological prop- erties. For the data in Figure 1(a), our algorithm found five distinct classes of beats, denoted in the figure by the arbitrary symbols $\theta, \gamma, \beta, \alpha$, and $\Psi$ (Figure $1(\mathrm{~b})$ ). For each class an archetypal beat is constructed that provides an easily understood visible representation of the types of beats in that class. The original ECG signal is then replaced by the corresponding sequence of symbols. This process allows us to shift from the analysis of raw signals to the analysis of symbolic strings. The discrete symbolic representation provides a layer of data reduction, reducing the data rate from $3960 \mathrm{bits} /$ second (sampling at $360 \mathrm{~Hz}$ with 11 bit quantization) to $n$ bits/second (where $n$ depends upon the number of bits needed to differentiate between symbols, three for this example). Finally, various techniques are used to find segments of the symbol sequence that are of potential clinical interest. In this example, a search for approximate repeating patterns found the rhythm shown in Figure 1(c). The corresponding archetypal representation in Figure 1(d) allows this activity to be readily visualized in a compact form.

The remainder of this paper is organized as follows. The process of symbolizing signals is described in Section 2 and the higher level analysis techniques that operate on this representation of the data in Section 3. An evaluation of our methods is presented alongside the technical details. A discussion of related work appears in Section 4, and a summary and conclusions are provided in Section 5. 


\section{SYMBOLIZATION}

An extensive literature exists on the subject of symbolization [4]. Essentially, the task of symbolizing data can be divided into two subtasks. As a first step, the signal needs to be segmented into intervals of activity. Following this, the set of segments is partitioned into classes and a label associated with each class.

The segmentation stage decomposes the continuous input signal into intervals with biologically relevant boundaries. A natural approach to achieve this is to segment the physiological signals according to some well-defined notion. In this work, we use R-R intervals for heart beats and peaks of inspiration and expiration for respiratory cycles. Since most cardiovascular signals are quasiperiodic, we can exploit cyclostationarity for data segmentation [5].

We treat the task of partitioning as a data clustering problem. Roughly speaking, the goal is to partition the set of segments into the smallest number of clusters such that each segment within a cluster represents the same underlying physiological activity. For example, in the case of ECG data, one cluster might contain only ventricular beats (i.e., beats arising from the ventricular cavities in the heart) and another only junctional beats (i.e., beats arising from a region of the heart called the atrioventricular junction). Each of these beats has different morphological characteristics that enable us to place them in different clusters.

There is a set of generally accepted labels that cardiologists use to differentiate distinct kinds of heart beats. Although cardiologists occasionally disagree about what label should be applied to some beats, labels supplied by cardiologists provide a useful way to check whether or not the beats in a cluster represent the same underlying physiological activity. However, in some cases, finer distinctions than provided by these labels can be clinically relevant. Normal beats, for example, are usually defined as beats that have morphologic characteristics that fall within a relatively broad range; for example, QRS complex less than 120 milliseconds and PR interval less than 200 milliseconds. Nevertheless, it may be clinically useful to further divide "normal" beats into multiple classes since some normal beats have subtle morphological features that are associated with clinically relevant states. One example of this phenomenon is Wolff-Parkinson-White (WPW) syndrome. In this disorder, patients have ECG beats that appear grossly normal, yet on close inspection, their QRS complexes contain a subtle deflection called a $\delta$-wave and a short PR interval [5]. Since such patients are predisposed to arrhythmias, the identification of this electrocardiographic finding is of interest [5]. For reasons such as this, standard labels cannot be used to check whether or not an appropriate number of clusters have been found.

We first extract features from each segment by sampling the continuous data stream at discrete points, and then group the segments based upon the similarity of their features. Many automated techniques exist for the unsupervised partitioning of a collection of individual observations into characteristic classes. In [6], a comprehensive examination of a number of methods that have been used to cluster ECG beats is provided. These methods focus on partitioning the beats into a relatively small number of well-documented classes. Our work differs both in our interest in making finer distinctions than is usual, for example, between two beats that would normally both be classified as "normal," and in our desire to discover classes that occur rarely during the course of a recording. This led us to employ clustering methods with a higher sensitivity than those described in [6]. In addition, we implement optimizations that facilitate the clustering of very large data sets.

We use Max-Min clustering to separate segmented units of cardiovascular signals into groups. The partitioning proceeds in a greedy manner, identifying a new group at each iteration that is maximally separated from existing groups and dynamic time-warping (DTW) is used to calculate the timenormalized distance between a pair of observations. This is described in Sections 2.1-2.2. An evaluation of this work is presented in Section 2.3.

\subsection{Dissimilarity metric}

Central to the clustering process is the method used to measure the distance between two segments. For physiological signals, this is complicated by the differences in lengths of segments. We deal with this using dynamic time-warping, which allows subsignals to be variably dilated or shrunk.

Given two segments $x_{1}$ and $x_{2}$, we measure the dissimilarity between them as the DTW cost of alignment [7]. Denoting the length of these sequences by $l_{1}$ and $l_{2}$, respectively, the conventional DTW algorithm produces the optimal alignment of the two sequences by first constructing an $l_{1}$-by- $l_{2}$ distance matrix. Each entry $(i, j)$ in this matrix represents the distance $d\left(x_{1}[i], x_{2}[j]\right)$ between samples $x_{1}[i]$ and $x_{2}[j]$. A particular alignment then corresponds to a path, $\varphi$, through the distance matrix of the form

$$
\varphi(k)=\left(\varphi_{1}(k), \varphi_{2}(k)\right), \quad 1 \leq k \leq K,
$$

where $\varphi_{1}$ and $\varphi_{2}$ represent row and column indices into the distance matrix, and $K$ is the alignment length.

The optimal alignment produced by DTW minimizes the overall cost:

$$
C\left(x_{1}, x_{2}\right)=\min _{\varphi} C_{\varphi}\left(x_{1}, x_{2}\right)
$$

with

$$
C_{\varphi}\left(x_{1}, x_{2}\right)=\frac{1}{K} \sum_{k=1}^{K} d\left(x_{1}\left[\varphi_{1}(k)\right], x_{2}\left[\varphi_{2}(k)\right]\right) .
$$

$C_{\varphi}$ is the total cost of path $\varphi$ divided by the alignment length, $K$. The division by $K$ is necessary since some long paths through the matrix will have large costs simply because they have more matrix elements. Dividing by $K$ helps to remove the dependence of the cost on the length of the original observations. The search for the optimal path then proceeds in $\mathrm{O}\left(l_{1} l_{2}\right)$ time by dynamic programming. One problem with this method is that some paths are long not because the segments to be aligned are long, but rather these observations 
are time-warped differently. In these cases, dividing by $K$ is inappropriate because the length of a beat (or of parts of a beat) being different often provides diagnostic information that is complimentary to the information provided by the morphology. Consequently, in our algorithm we omit the division by $K$.

Another important difference between our approach and traditional DTW is the distance metric used. The conventional DTW algorithm defines the distance $d\left(x_{1}[i], x_{2}[j]\right)$ as the Euclidean distance between the individual samples $x_{1}[i]$ and $x_{2}[j]$. In the presence of small amounts of additive background noise, similar to what is commonly encountered in physiological signals, a more robust measure is provided by calculating the distance between small windows of the signals $x_{1}$ and $x_{2}$, centred at time instants $i$ and $j$, that is,

$$
d\left(x_{1}[i], x_{2}[j]\right)=\left(\frac{1}{2 W+1} \sum_{k=-W}^{W}\left(x_{1}[i+k]-x_{2}[j+k]\right)\right)^{2} .
$$

The key idea is that the distance is computed across local windows to better capture underlying trends, as opposed to individual samples, which are more sensitive to noise. $W$ is typically chosen to be a small value depending on the sampling frequency so as to prevent the possibility of sharp events such as the QRS complex from being diminished in amplitude. For these studies we chose $W=4$, a compromise between the need to remove background noise and the need to preserve important morphologic characteristics of the signal.

Essentially, this approach is equivalent to first smoothing out the signals $x_{1}$ and $x_{2}$ by median filtering with a small window of length $2 W+1$, and may be carried out with a subsequent preprocessing step. We recognize that other methods for removing background noise exist [8], and future applications of this work will explore these alternate approaches.

\subsection{Max-Min clustering}

In $[9,10]$, clustering methods are proposed that build on top of the dissimilarity measure presented in Section 2.1. A modified fuzzy clustering approach is described in [9], while [10] explores the use of hierarchical clustering. Denoting the number of observations to be clustered as $N$, both methods require a total of $\mathrm{O}\left(N^{2}\right)$ comparisons to calculate the dissimilarity between every pair of observations. If each observation has length $M$, the time taken for each dissimilarity comparison is $\mathrm{O}\left(M^{2}\right)$. Therefore, the total running time for the clustering methods in $[9,10]$ is $\mathrm{O}\left(M^{2} N^{2}\right)$. Additionally, storing the entire matrix of comparisons between every pair of observations requires $\mathrm{O}\left(N^{2}\right)$ space.

To reduce the requirements in terms of running time and space, we employ Max-Min clustering [11], which can be implemented to discover $k$ clusters using $\mathrm{O}(N k)$ comparisons. This leads to a total running time of $\mathrm{O}\left(M^{2} N k\right)$, with an $\mathrm{O}(N)$ space requirement.

Max-Min clustering proceeds by choosing an observation at random as the first centroid $c_{1}$ and setting the set $S$ of centroids to $\left\{c_{1}\right\}$. During the $i$ th iteration, $c_{i}$ is chosen such that it maximizes the minimum distance between $c_{i}$ and observations in $S$ :

$$
c_{i}=\arg \max _{x \notin S} \min _{y \in S} C(x, y),
$$

where $C(x, y)$ is defined as in (2). The set $S$ is incremented at the end of each iteration such that $S=S \cup c_{i}$.

The number of clusters discovered by Max-Min clustering is chosen by iterating until the maximized minimum dissimilarity measure in (5) falls below a specified threshold $\theta$. Therefore, the number of clusters, $k$, depends on the separability of the underlying data to be clustered.

The running time of $\mathrm{O}\left(M^{2} N k\right)$ can be further reduced by exploiting the fact that in many cases two observations may be sufficiently similar that it is not necessary to calculate the optimal alignment between them. A preliminary processing block that identifies $c$ such homogeneous groups from $N$ observations without alignment of time-samples will reduce the number of DTW comparisons, each of which is $\mathrm{O}\left(M^{2}\right)$, from $\mathrm{O}(N k)$ to $\mathrm{O}(c k)$. This preclustering can be achieved in a computationally inexpensive manner through an initial round of Max-Min clustering using a simple distance metric.

The running time using preclustering is given by $\mathrm{O}(M N c)+\mathrm{O}\left(M^{2} c k\right)$. The asymptotic worst case behavior with this approach is still $\mathrm{O}\left(M^{2} N k\right)$, for example, when all the observations are sufficiently different that $c=N$. However, for the ECG data we have examined, $c$ is an order of magnitude less than $N$. For example, preclustering with a hierarchical Max-Min approach yielded a speedup factor of 12 on the data from the MIT-BIH arrhythmia database used for the work described in Section 2.3.

\subsection{Evaluation of clustering algorithm}

We applied the techniques discussed in Sections 2.1-2.2 to electrocardiographic data in the Physionet MIT-BIH Arrhythmia database, which contains excerpts of two-channel ECG sampled at $360 \mathrm{~Hz}$ per channel with 11-bit resolution. Activity is hand-annotated by cardiologists, allowing our findings to be validated against human specialists.

For each patient in the database, we searched for different classes of ECG activity between consecutive R waves within each QRS complex. A Max-Min threshold of $\theta=50$ was used, with this value being chosen experimentally to produce a small number of clusters, while generally separating out clinical classes of activity for each patient. As we report at the end of this section, a prospective study on blind data not used during the original design of our algorithm shows that the value of the $\theta$ parameter generalizes quite well.

Beats were segmented using the algorithm described in [12]. A histogram for the number of clusters found automatically for each patient is provided in Figure 2. The median number of clusters per patient was 22. For the median patient, 2202 distinct beats were partitioned into 22 classes. A relatively large number of clusters were found in some 


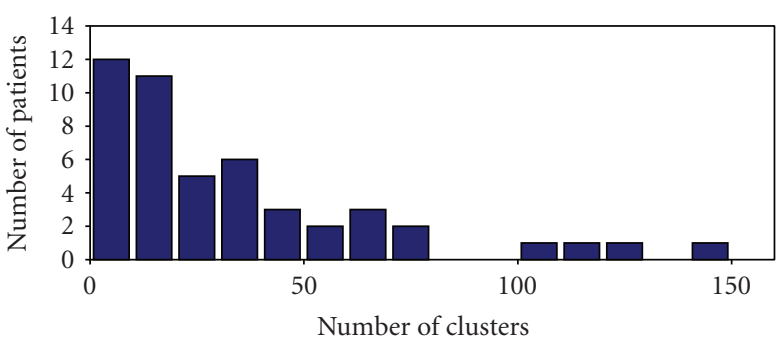

FIGURE 2: Histogram of clusters per patient: the number of clusters determined automatically per patient is distributed as shown, with a median value of 22 .

cases, in particular patients $105,203,207$, and 222 . These files are described in the MIT-BIH Arrhythmia database as being difficult to analyze owing to considerable high-grade baseline noise and muscle artifact noise. This leads to highly dissimilar beats, and also makes the ECG signals difficult to segment. For patient 207, the problem is compounded by the presence of multiform premature ventricular contractions (PVCs). Collectively, these records are characterized by long runs of beats corresponding to singleton clusters, which can be easily detected and discarded (i.e., long periods of time where every segmented unit looks significantly different from everything else encountered).

Our algorithm clusters data without incorporating prior, domain-specific knowledge. As such, our method was not designed to solve the classification problem of placing beats into prespecified clinical classes corresponding to cardiologist labels. Nevertheless, a comparison between our clustering algorithm and cardiologist provided labels is of interest. Therefore, we compared our partitioning of the data to cardiologist-provided labels included in the MIT-BIH arrhythmia database.

There are a number of ways to compare a clustering produced by our algorithm $\left(C_{A}\right)$ to the implicit clustering which is defined by cardiologist supplied labels $\left(C_{L}\right) . C_{A}$ and $C_{L}$ are said to be isomorphic if for every pair of beats, the beats are in the same cluster in $C_{A}$ if and only if they are in the same cluster in $C_{L}$. If $C_{A}$ and $C_{L}$ are isomorphic, our algorithm has duplicated the clustering provided by cardiologists. In most cases, $C_{A}$ and $C_{L}$ will not be isomorphic because our algorithm typically produces more clusters than are traditionally defined by cardiologists. We view this as an advantage of our approach as it enables our method to identify new morphologies and patterns that may be of clinical interest.

Alternatively, we say that $C_{A}$ is consistent with $C_{L}$ if an isomorphism between the two can be created by merging clusters in $C_{A}$. For example, two beats in an ECG data stream may have abnormally long lengths and therefore represent "wide-complex" beats. However, if they have sufficiently different morphologies, they will be placed in different clusters. We can facilitate the creation of an isomorphism between $C_{A}$ and $C_{L}$ by merging all clusters in $C_{A}$ which consists of widecomplex beats. While consistency is a useful property, it is not sufficient. For example, if every cluster in $C_{A}$ contained exactly one beat, it would be consistent with $C_{L}$. As discussed above, however, in most cases our algorithm produces a reasonable number of clusters.

To determine whether our algorithm generates a clustering that is consistent with cardiologists supplied labels, we examined the labels of beats in each cluster and assigned the cluster a label corresponding to its majority element. For example, a cluster containing 1381 normal beats, and 2 atrial premature beats would be labeled as being normal. Beats in the original signal were then assigned the labels of their clusters (e.g., the 2 atrial beats in the above example would be labeled as normal). Finally, we tabulate the differences between the labels generated by this process and the cardiologist supplied labels in the database. This procedure identifies, and effectively merges, clusters that contain similar types of beats.

We considered only classes of activity that occurred in at least $5 \%$ of the patients in the population, that is, 3 or more patients in the MIT-BIH Arrhythmia database. Specifically, even though we successfully detected the presence of atrial escape beats in patient 223 of the MIT-BIH Arrhythmia database and ventricular escape beats in patient 207, we do not report these results in the subsequent discussion since no other patients in the population had atrial or ventricular escape activity and it is hard to generalize from performance on a single individual. During the evaluation process, labels that occur fewer than three times in the original labeling for a patient (i.e, less than $0.1 \%$ of the time) were also ignored.

Tables 1 and 2 show the result of this testing process. We document differences between the labeling generated by our process and the cardiologist supplied labels appearing in the database. Differences do not necessarily represent errors. Visual inspection of these differences by a board-certified cardiologist, who was not involved in the initial labeling of beats in the Physionet MIT-BIH arrhythmia database, indicates that experts can disagree on the appropriate labeling of many of the beats where the classification differed. Nevertheless, for simplicity we will henceforth refer to "differences" as "errors."

In Table 1, for the purpose of compactly presenting results, we organize clinical activity into the following groups:

(i) normal;

(ii) atrial (atrial premature beats, aberrated atrial premature beats and atrial ectopic beats);

(iii) ventricular (premature ventricular contractions, ventricular ectopic beats, and fusion of normal and ventricular beats);

(iv) bundle branch block (left and right bundle branch block beats);

(v) junctional (premature junctional beats and junctional escape beats);

(vi) others.

The result of clustering without this grouping (i.e., in terms of the original annotations in the MIT-BIH Arrhythmia database) is presented in Table 4 . The overall misclassification percentage in both cases is approximately $1.4 \%$. 
TABLE 1: Beats detected for each patient in the MIT-BIT Arrhythmia database using symbolization. To compactly display results we group the clinical classes $(\mathrm{N}=$ normal, Atr. = atrial arrhythmias, Ven. = ventricular, Bbb. = bundle branch block, Jct. = junctional beats, Oth. = others, Mis. = mislabeled beat). For each group, the number of correctly detected beats is shown relative to the total beats originally present. The aggregate detection performance is given in terms of both beats (i.e., total number of beats for each group correctly detected across population) and patients (i.e., total number of patients for whom the group of activity was correctly detected to occur).

\begin{tabular}{|c|c|c|c|c|c|c|c|c|}
\hline Patient & $\mathrm{N}$ & Atr. & Ven. & Bbb. & Jct. & Oth. & Mis. & Mis. $\%$ \\
\hline 100 & $2234 / 2234$ & $30 / 33$ & - & - & - & - & $3 / 2267$ & $0.13 \%$ \\
\hline 101 & $1852 / 1852$ & $3 / 3$ & - & - & - & - & $0 / 1855$ & $0.00 \%$ \\
\hline 102 & $14 / 99$ & - & $4 / 4$ & - & - & $2077 / 2079$ & $87 / 2182$ & $3.99 \%$ \\
\hline 103 & $2076 / 2076$ & - & - & - & - & - & 0/2076 & $0.00 \%$ \\
\hline 104 & $51 / 163$ & - & - & - & - & $2027 / 2040$ & $125 / 2203$ & $5.67 \%$ \\
\hline 105 & $2530 / 2534$ & - & $39 / 40$ & - & - & - & $5 / 2574$ & $0.19 \%$ \\
\hline 106 & $1500 / 1500$ & - & $508 / 511$ & - & - & - & $3 / 2011$ & $0.15 \%$ \\
\hline 107 & - & - & $59 / 59$ & - & - & $2074 / 2075$ & $1 / 2134$ & $0.05 \%$ \\
\hline 108 & $1748 / 1748$ & $1 / 4$ & $17 / 18$ & - & - & - & $4 / 1770$ & $0.23 \%$ \\
\hline 109 & - & - & $37 / 40$ & $2486 / 2486$ & - & - & $3 / 2526$ & $0.12 \%$ \\
\hline 111 & $2117 / 2117$ & - & - & - & - & - & $0 / 2117$ & $0.00 \%$ \\
\hline 112 & $2533 / 2533$ & - & - & - & - & - & $0 / 2533$ & $0.00 \%$ \\
\hline 113 & $1782 / 1782$ & $5 / 5$ & - & - & - & - & $0 / 1787$ & $0.00 \%$ \\
\hline 114 & $1815 / 1815$ & $4 / 8$ & $47 / 48$ & - & - & - & $5 / 1871$ & $0.27 \%$ \\
\hline 115 & $1946 / 1946$ & - & - & - & - & - & $0 / 1946$ & $0.00 \%$ \\
\hline 116 & $2281 / 2281$ & - & $107 / 107$ & - & - & - & $0 / 2388$ & $0.00 \%$ \\
\hline 117 & $1528 / 1528$ & - & - & - & - & - & $0 / 1528$ & $0.00 \%$ \\
\hline 118 & - & $82 / 96$ & $16 / 16$ & $2147 / 2161$ & - & - & $28 / 2273$ & $1.23 \%$ \\
\hline 119 & $1540 / 1540$ & - & $443 / 443$ & - & - & - & 0/1983 & $0.00 \%$ \\
\hline 121 & $1858 / 1858$ & - & - & - & - & - & $0 / 1858$ & $0.00 \%$ \\
\hline 122 & $2475 / 2475$ & - & - & - & - & - & $0 / 2475$ & $0.00 \%$ \\
\hline 123 & $1510 / 1510$ & - & - & - & - & - & $0 / 1510$ & $0.00 \%$ \\
\hline 124 & - & - & $52 / 52$ & $1523 / 1526$ & $6 / 34$ & - & $31 / 1612$ & $1.92 \%$ \\
\hline 200 & $1737 / 1739$ & $1 / 29$ & $796 / 815$ & - & - & - & $49 / 2583$ & $1.90 \%$ \\
\hline 201 & $1605 / 1605$ & $65 / 76$ & $184 / 185$ & - & $3 / 11$ & - & $20 / 1877$ & $1.07 \%$ \\
\hline 202 & $2043 / 2046$ & $32 / 48$ & $18 / 20$ & - & - & - & $21 / 2114$ & $0.99 \%$ \\
\hline 203 & $2432 / 2442$ & - & $318 / 345$ & - & - & - & $37 / 2787$ & $1.33 \%$ \\
\hline 205 & $2564 / 2565$ & $1 / 3$ & 76/77 & - & - & - & $4 / 2645$ & $0.15 \%$ \\
\hline 207 & - & $114 / 116$ & $190 / 208$ & $1538 / 1559$ & - & - & $41 / 1883$ & $2.18 \%$ \\
\hline 208 & $1507 / 1575$ & - & $1327 / 1348$ & - & - & - & $89 / 2923$ & $3.04 \%$ \\
\hline 209 & $2603 / 2617$ & $317 / 383$ & - & - & - & - & $80 / 3000$ & $2.67 \%$ \\
\hline 210 & $2411 / 2416$ & $14 / 21$ & $164 / 183$ & - & - & - & $31 / 2620$ & $1.18 \%$ \\
\hline 212 & $920 / 920$ & - & - & $1821 / 1824$ & - & - & $3 / 2744$ & $0.11 \%$ \\
\hline 213 & $2632 / 2635$ & $4 / 28$ & $321 / 581$ & - & - & - & $287 / 3244$ & $8.85 \%$ \\
\hline 214 & - & - & $260 / 261$ & $1980 / 1993$ & - & - & $14 / 2254$ & $0.62 \%$ \\
\hline 215 & $3190 / 3191$ & - & $156 / 159$ & - & - & - & $4 / 3350$ & $0.12 \%$ \\
\hline 217 & $229 / 242$ & - & $138 / 157$ & - & - & $1720 / 1802$ & $114 / 2201$ & $5.18 \%$ \\
\hline 219 & $2077 / 2077$ & $0 / 7$ & $31 / 63$ & - & - & - & $39 / 2147$ & $1.82 \%$ \\
\hline 220 & $1942 / 1947$ & $91 / 93$ & - & - & - & - & $7 / 2040$ & $0.34 \%$ \\
\hline 221 & $2028 / 2028$ & - & $381 / 382$ & - & - & - & $1 / 2410$ & $0.04 \%$ \\
\hline 222 & $1939 / 1977$ & $121 / 187$ & - & - & $125 / 216$ & - & $195 / 2380$ & $8.19 \%$ \\
\hline 223 & $2021 / 2025$ & 20/89 & $462 / 484$ & - & - & - & $95 / 2598$ & $3.66 \%$ \\
\hline 228 & $1685 / 1687$ & $0 / 3$ & $366 / 371$ & - & - & - & $10 / 2061$ & $0.49 \%$ \\
\hline 230 & $2249 / 2249$ & - & - & - & - & - & $0 / 2249$ & $0.00 \%$ \\
\hline 231 & $312 / 312$ & - & - & $1246 / 1247$ & - & - & $1 / 1559$ & $0.06 \%$ \\
\hline 232 & - & $1407 / 1423$ & - & $435 / 437$ & - & - & $18 / 1860$ & $0.97 \%$ \\
\hline 233 & $2219 / 2220$ & $0 / 7$ & $814 / 828$ & - & - & - & $22 / 3055$ & $0.72 \%$ \\
\hline 234 & $2695 / 2696$ & - & $3 / 3$ & - & $35 / 50$ & - & $16 / 2749$ & $0.58 \%$ \\
\hline Total beats & $76430 / 76802$ & $2312 / 2662$ & $7334 / 7808$ & $13176 / 13233$ & $169 / 311$ & $7898 / 7996$ & $1493 / 108812$ & $1.37 \%$ \\
\hline Total patients & $41 / 41$ & $18 / 21$ & $29 / 29$ & $8 / 8$ & $4 / 4$ & $4 / 4$ & - & - \\
\hline
\end{tabular}


TABle 2: Summary comparison of detection through symbolization to cardiologist supplied labels. The labels used correspond to the original MIT-BIH Arrhythmia database annotations $(\mathrm{N}=$ normal, $\mathrm{L}=$ left bundle branch block, $\mathrm{R}=$ right bundle branch block, $\mathrm{A}=$ atrial premature beats, $\mathrm{a}=$ aberrated atrial premature beats, $\mathrm{V}=$ premature ventricular complex, $\mathrm{P}=$ paced beat, $\mathrm{f}=$ fusion of normal and paced beat, $F=$ fusion of ventricular and normal beat, $j=$ junctional escape beat). The top row is indicative of how well the clustering did at identifying the presence of classes of clinical activity identified by the cardiologists for each patient. The bottom row indicates how well the clustering did at assigning individual beats to the same classes as the cardiologists.

\begin{tabular}{|c|c|c|c|c|c|c|c|c|c|c|}
\hline & $\mathrm{N}$ & $\mathrm{L}$ & $\mathrm{R}$ & A & a & $\mathrm{V}$ & $\mathrm{P}$ & $\mathrm{f}$ & $\mathrm{F}$ & $\mathrm{j}$ \\
\hline $\begin{array}{l}\text { Percentage of total } \\
\text { patients detected }\end{array}$ & 100.0 & 100.00 & 100.00 & 84.21 & 100.00 & 100.00 & 100.00 & 100.00 & 75.00 & 100.00 \\
\hline $\begin{array}{l}\text { Percentage of total } \\
\text { beats detected }\end{array}$ & 99.52 & 99.50 & 99.67 & 87.30 & 85.11 & 96.80 & 99.91 & 78.75 & 46.69 & 56.96 \\
\hline
\end{tabular}

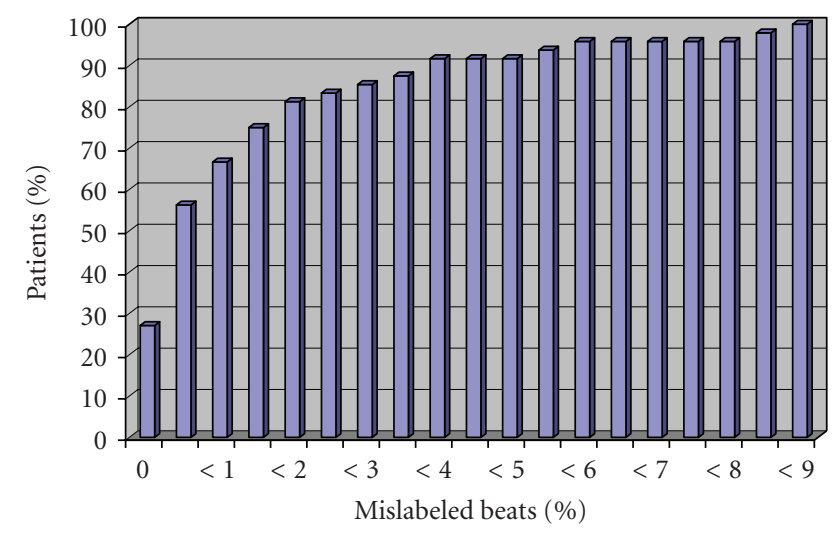

Figure 3: Mislabeling error: over a quarter of the patients had no mislabeling errors using our clustering approach, over $65 \%$ had less than $1 \%$ mislabeled beats relative to cardiologist labels.

Figure 3 also illustrates how the mislabeling error associated with our clustering approach is distributed across patients. In the majority of the patients, there is less than $1 \%$ error.

As Tables 1 and 2 indicate, our symbolization technique does a reasonably good job both at identifying clinically relevant clusters and at assigning individual beats to the appropriate cluster.

The data in the first row of Table 2 sheds light on critical errors, that is, errors that cause one to conclude that a patient does not exhibit a certain type of beat when, in fact, their ECG signal does contain a significant number of the beats in question. More precisely, we say that a critical error has occurred when a patient has at least three instances of a clinically relevant type of beat and there does not exist at least one cluster in which that beat is a majority element. For example, for each patient for whom the cardiologists found three or more "premature ventricular complexes," the algorithm formed a cluster for beats of that type. On the other hand, for one quarter of the patients with at least three "fusion of ventricular and normal beats," the algorithm did not form a cluster for that type of beat.

In 43 out of 48 patients there were no critical errors. This is important because, in the presence of critical errors, an

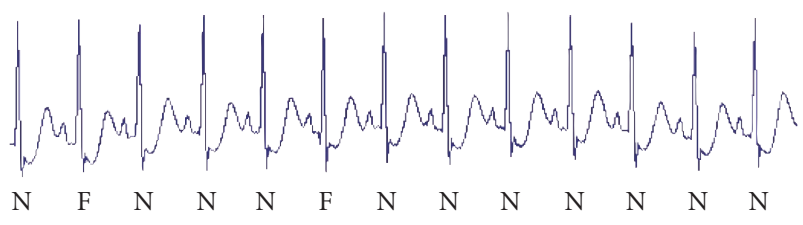

Figure 4: Raw tracing of ECG for patient 213 in the MIT-BIH database with fusion of ventricular and normal beats: a sequence of ECG is shown containing beats labeled as both normal $(\mathrm{N})$ and fusion $(\mathrm{F})$. The morphological differences between the two classes of beats are subtle. This excerpt corresponds to time $4: 15$ in the recording.

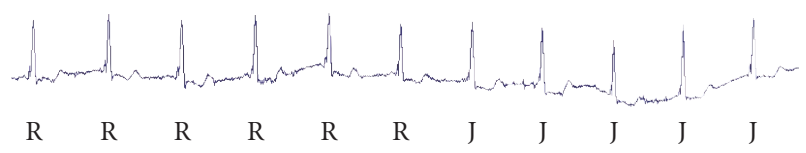

FIGURE 5: Raw tracing of ECG for patient 124 in the MIT-BIH database with junctional escape beats: a sequence of ECG is shown containing both right bundle branch block $(\mathrm{R})$ and junctional premature $(\mathrm{J})$ beats. The morphological differences between the two classes of beats are again subtle. This excerpt corresponds to time $4: 39$ in the recording.

inspection of the data through visualization of the cluster representatives would conceal the presence of some activity in the dataset. Avoiding critical errors is a challenge, because for some patients, the number of elements in different clinical classes varies by a few orders of magnitude. For example, as can be seen in the appendix, for patient 101, the process correctly identifies the three atrial premature beats amidst the 1852 normal beats.

For some classes of activity, however, our morphologybased clustering generated labels different from those provided by the cardiologists. Figure 4 presents an example where morphology-based clustering differed from the labels in the database. However, given the similarity between the beats labeled $\mathrm{F}$ and $\mathrm{N}$ in the database, it is not clear that our algorithm is in error. Similarly, our algorithm also failed to distinguish right bundle branch block and junctional premature beats, as shown in Figure 5. 


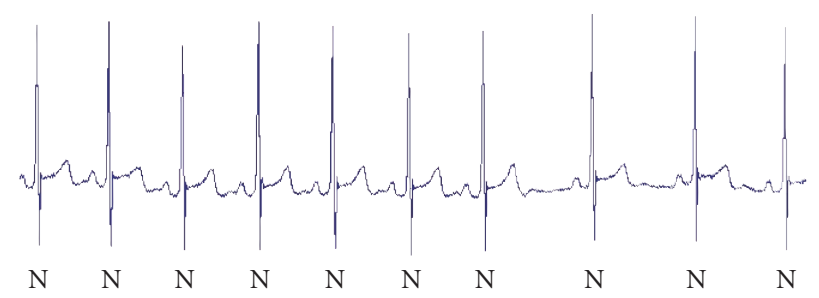

FIGURE 6: Raw tracing of ECG for patient 115 in the MIT-BIH database with normal beats: a sequence of ECG is shown containing normal beats. This sequence represents an example where morphology-based analysis separates the beats into short (first 7 beats) and long (last three beats) classes. The beats still fall in the same clinical class, but this separation, which indicates an abrupt change in heart rate, may potentially be of interest for the purpose of higher level analysis. This excerpt corresponds to time $7: 40$ in the recording.

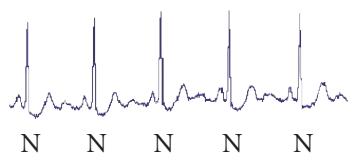

(a)

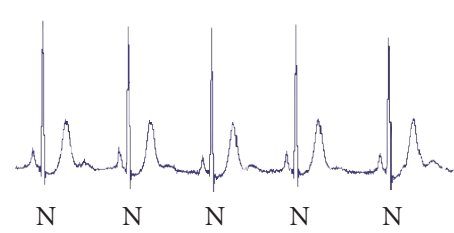

(b)

FIgURE 7: Raw tracing of ECG for patient 106 in the MIT-BIH database with normal beats: (a) ECG corresponding to time 16:54 in the file. (b) ECG corresponding to time 21:26 in the file. Morphology-based analysis places the beats shown in (a) and (b) into separate clusters based on changes in amplitude.

Sometimes our algorithm places beats for which cardiologists have supplied the same label into different clusters. As was discussed above, this is not necessarily a bad thing as subtle distinctions between "normal" beats may contain useful clinical information. Figures 6 and 7 present instances in which our algorithm separated beats that were assigned the same label by cardiologists. In Figure 6, morphologybased analysis is able to distinguish changes in length. In Figure 7, changes in amplitude are discerned automatically. These morphological differences may represent clinically important distinctions. In each instance, beats which are classified as "normal" have very different morphologic features that may be associated with important disease states. Abrupt changes in the R-R interval, like that noted in Figure 6, correspond to rapid fluctuations in the heart-a finding which can be associated with a number of clinically important conditions such as Sick sinus Syndrome (SSS) or sinus arrhythmia [5]. Similarly, significant changes in QRS amplitude, like that seen in Figure 7, can be observed in
TABLE 3: Summary comparison of detection through symbolization to cardiologist supplied labels for the MGH/MF waveform database. The labels of the columns match those in Table 2 with $\mathrm{J}=$ junctional premature beats.

\begin{tabular}{l|ccccc}
\hline & $\mathrm{N}$ & $\mathrm{V}$ & $\mathrm{P}$ & $\mathrm{J}$ & $\mathrm{F}$ \\
\hline $\begin{array}{l}\text { Percentage of total } \\
\text { clust. detected }\end{array}$ & 100.00 & 100.00 & 100.00 & 100.00 & 100.00 \\
$\begin{array}{l}\text { Percentage of total } \\
\text { beats detected }\end{array}$ & 99.91 & 96.51 & 98.84 & 100.0 & 100.0 \\
\hline
\end{tabular}

patients with large pericardial effusions [5]. Both of these diagnoses are important syndromes that can be associated with adverse clinical outcomes. Therefore, we view the ability to make such distinctions between beats as a benefit of the method.

Data from the MIT-BIH arrhythmia database were used during the initial design of the symbolization algorithm, and the results reported in Tables 1 and 2 were generated on this data set. To test the robustness of the method, we also tested our algorithm on ECG data on the first forty patients from the MGH/MF waveform database (i.e., mgh001-mgh040), which was not used in design of the algorithm. This dataset contains fewer episodes of interesting arrhythmic activity than the MIT-BIH arrhythmia database and is also relatively noisy, but contains ECG signals sampled at the same rate (i.e., $360 \mathrm{~Hz}$ ) with 12 -bit resolution, that is, a sampling rate and resolution similar to that of the MIT-BIH arrhythmia database. The recordings are also typically an hour long instead of 30 minutes for the MIT-BIH arrhythmia database. Table 3 shows the performance of the symbolization algorithm on this dataset. The results are comparable to the ones obtained for the MIT-BIH arrhythmia dataset.

The median number of clusters found in this case was 43 . We removed file mgh026 from analysis because of the many errors in the annotation file which prevented any meaningful comparisons against the cardiologist-provided labels. We also removed file mgh002, which was corrupted by noise that led to errors in the segmentation of the ECG signal. We also detected the presence of atrial escape beats for patient mgh018, but do not report results for this class in Table 3 since no other patients revealed similar activity.

\section{HIGHER LEVEL ANALYSES}

Symbolization leads to a discrete representation of the original cardiovascular signals. The goal of this analysis is to develop techniques that operate on these symbolic data to discover subsequences that correspond to clinically relevant activity in the original signal. A key aspect of our approach is that no domain expertise is used to identify subsequences in the original data stream.

Since our intent is to apply these techniques to massive data sets, computational efficiency is an important consideration. The techniques also need to operate robustly on noisy symbolic signals. There are two important sources of noise, noisy sensors and imperfections in the symbolization 
process, that assign distinct symbols to beats that should have been assigned the same symbol.

In this section, we present two classes of techniques: techniques designed to extract relevant information from individual signals (Section 3.1); and techniques designed to extract relevant information across multiple signals (Section 3.2). We evaluate the techniques in Section 3.3. We provide examples showing that the techniques can indeed be used to find segments of the original signal (or signals) that correspond to activity described by cardiologists as clinically relevant. We would have liked to perform a quantitative analysis of sensitivity and specificity. However, since we were unable to find a public domain database in which all of the events in the signals were marked (e.g., correlation amongst signals, the presence of rhythms such as cardiac ballet, etc.), such an analysis was not carried out.

\subsection{Analyzing single signal streams}

In this section, we examine ways for finding rhythms, recurrent transient patterns, and segments with high or low entropy in a single data stream.

\subsubsection{Rhythms}

A sequence $w_{1} w_{2} \cdots w_{H}$ constitutes an exact or perfect repeat in a symbolic signal $v_{1} v_{2} \cdots v_{N}$ with $L>1$ periods if for some starting position $s$,

$$
v_{s} v_{s+1} \cdots v_{s+H L-1}=\left(w_{1} w_{2} \cdots w_{H}\right)^{L} .
$$

The number of repeating periods $L$ can be chosen to trim the set of candidate repeats. We define rhythms as repeating subsequences in a symbolic signal. To address the issue of noise, we generalize the notion in (6) to approximate repeats, which allow for mismatches between adjacent repeats. A sequence $w_{1} w_{2} \cdots w_{H}$ is an approximate repeat with $L$ periods if there exists a set of strictly increasing positions $s_{1}, \ldots, s_{L+1}$ such that for all $1 \leq i \leq L$,

$$
\varphi\left(w_{1} w_{2} \cdots w_{H}, v_{s_{i}} v_{s_{i}+1} \cdots v_{s_{i+1}-1}\right) \leq \gamma,
$$

where $\phi(p, q)$ represents a measure of the distance between sequences $p$ and $q$ (e.g., the Hamming distance [13]) and $\gamma$ is a threshold constraining the amount of dissimilarity allowed across the repeats. The final position $s_{L+1}$ can be at most one more than the length of $v_{1} v_{2} \cdots v_{N}$.

The problem of detecting all approximate repeats in a symbolic signal can be solved using the algorithm presented in [14] with a running time of $\mathrm{O}(N \gamma a \log (N / \gamma))$, where $a$ corresponds to the maximum number of periods in the signal. Examples of clinical conditions that can be detected by this approach are bigeminy, trigeminy, and heart block.

\subsubsection{Recurrent transient patterns}

A related problem to detecting rhythms is detecting short recurrent patterns. These subsequences may be comprised of repeats that are not sustained long enough to be discovered by the techniques in Section 3.1.1.
The mining of physiological signals for recurrent transient patterns can be mapped to the task of detecting statistically significant subsequences that occur with sufficient frequency. The challenge is to discover complexes $w_{1} w_{2} \cdots w_{H}$ with shared spatial arrangement that occur more frequently in the symbolic signal $v_{1} v_{2} \cdots v_{N}$ than would be expected given the background distribution over the symbols in the data. The ranking function for this criterion considers two factors: (1) the significance of a pattern relative to the background distribution of symbols; and (2) the absolute count of the number of times the pattern was observed in the data stream. Denoting the probability operator by Pr, the first criterion is equivalent to evaluating the expression

$$
\frac{\operatorname{Pr}\left(w_{1} w_{2} \cdots w_{H}\right)}{\prod_{i=1}^{H} \operatorname{Pr}\left(w_{i}\right)} .
$$

The second criterion is necessary to deal with situations where the pattern contains a very rare symbol. Depending on the length of the pattern, the probability ratio in (8) may be unduly large in such instances. Hence, the absolute number of times that the pattern occurs is explicitly considered. Exact patterns that occur with high frequency can be found by a linear traversal of $v_{1} v_{2} \cdots v_{N}$ while maintaining state to record the occurrence of each candidate pattern. Inexact patterns can be handled by searching in the neighborhood of a candidate pattern in a manner similar to BLAST [15].

An example of a clinical condition that can be detected by this approach is paroxysmal atrial tachycardia.

\subsubsection{Entropy}

Short bursts of irregular activity can be detected by searching for episodes of increased entropy. We search for subsequences in symbolic signals with an alphabet of size $\Lambda$ in which the entropy approaches $\log _{2} \Lambda$. An example of a clinical condition that can be detected by this approach is atrial fibrillation.

Conversely, the absence of sufficient variation (e.g., changes in the length of heart beats arising due to natural fluctuations in the underlying heart rate) can be recognized by the lack of entropy over long time scales.

\subsection{Multisignal trends}

The presence of massive datasets restricts visibility of multimodal trends. Most humans are restricted in their ability to reason about relationships between more than two inputs [16]. Automated systems can help address this limitation, but techniques to analyze raw time-series data are computationally intensive, particularly for signals with high sampling rates. Mutual information analysis cannot readily be applied to raw data, particularly in the presence of time warping. As shown in [17] (see Section 4), the symbolic representation of the signal can greatly simplify this problem.

For example, one can examine the mutual information across $M$ sequences of symbols by treating each sequence as a random variable $V_{i}$, for $1 \leq i \leq M$, and examining the 
multivariate mutual information $\mathrm{I}\left(V_{1}, \ldots, V_{M}\right)[18]$ :

$$
\sum_{j=1}^{M} \sum_{\left\{i_{1}, \ldots, i_{j}\right\} \subseteq\{1, \ldots, M\}}(-1)^{j+1} H\left(V_{i_{1}}, \ldots, V_{i_{j}}\right),
$$

where $H$ denotes the joint entropy between random variables. Computing $\mathrm{I}\left(V_{1}, \ldots, V_{M}\right)$ in this manner is intractable for large values of $M$. For computational efficiency, it is possible to employ $k$-additive truncation [19], which neglects corrective or higher order terms of order greater than $k$.

An alternative formulation of the problem of detecting multimodal trends involves assessing the degree of association of sequences in $M$ with activity in a sequence not in $M$ (denoted by $V_{\text {NEW }}$ ). Consider a set of symbols $U_{i}$, each corresponding to a realization of the random variable $V_{i}$, for 1 $\leq i \leq M$. Let $H\left(V_{\text {NEW }}^{\tau}\right)$ be the entropy in $V_{\text {NEW }}$ at all time instants $t$ that are some specified time-lag, $\tau$, away from each joint occurrence of the symbols $U_{i}$. That is, $H\left(V_{\text {NEW }}^{\tau}\right)$ measures the entropy in $V_{\text {NEW }}$ at all time instants $t$ satisfying the predicate

$$
\left(V_{1}[t-\tau]=U_{1}\right) \wedge \cdots \wedge\left(V_{M}[t-\tau]=U_{M}\right) .
$$

We then define the time-lagged association between the joint occurrence of the symbols $U_{i}$ and signal $V_{\mathrm{NEW}}$ as

$$
H\left(V_{\mathrm{NEW}}\right)-H\left(V_{\mathrm{NEW}}^{\tau}\right) \text {. }
$$

If a time-lagged association exists, the entropy in $V_{\mathrm{NEW}}$ at all time instants $t$ that obey the predicate in (10) will be less than the entropy across the entire signal, that is, activity at these time instants will be more predictable and consistent with the underlying event in signals $V_{1}$ through $V_{M}$.

The difference between the formulations described by (9) and (11) can be appreciated by considering two signals $V_{1}$ and $V_{2}$. Equation (9) essentially determines if the two are correlated. In (11), the focus is on identifying whether a specific class of activity in $V_{1}$ is associated with a consistent event in $V_{2}$, even if the signals may otherwise be uncorrelated. Figure 8 indicates the differences. Searching for timelagged associations using the method in (11) is likely to be important for discovering activity that is associated with clinical events.

An example of a clinical condition that can be detected by this approach is pulsus paradoxus.

\subsection{Evaluation of symbolic analysis}

The techniques for single-signal analysis discussed in Section 3.2 were tested on the MIT-BIH arrhythmia database.

\subsubsection{Analysis of single ECG signals}

Figures 9 and 10 provide examples of applying the approximate repeat detection techniques described in Section 3.1. The figures show a fragment of the raw signal and a pictorial representation of the symbol stream for that fragment. The pictorial representation provides a compact display of

\begin{tabular}{|c|c|c|c|c|c|c|c|}
$\mathrm{X}$ & $\mathrm{Y}$ & $\mathrm{Y}$ & $\mathrm{Y}$ & $\mathrm{X}$ & $\mathrm{Y}$ & $\mathrm{Y}$ & $\mathrm{Y}$ \\
$\mathrm{A}$ & $\mathrm{B}$ & $\mathrm{B}$ & $\mathrm{B}$ & $\mathrm{A}$ & $\mathrm{B}$ & $\mathrm{B}$ & $\mathrm{B}$ \\
$\mathrm{A}$ & $\mathrm{B}$ & $\mathrm{C}$ & $\mathrm{D}$ & $\mathrm{A}$ & $\mathrm{B}$ & $\mathrm{C}$ & $\mathrm{D}$
\end{tabular}

(a) Traditional correlation

$\begin{array}{llllllll}\text { X } & \text { Y } & \text { Y } & \text { Y } & \text { X } & \text { Y } & \text { Y } & \text { Y } \\ \text { A } & \text { B } & \text { B } & \text { B } & \text { A } & \text { B } & \text { B } & \text { B } \\ \text { A } & \text { B } & \text { C } & \text { D } & \text { A } & \text { B } & \text { C } & \text { D }\end{array}$

(b) Time-lagged association

FIGURE 8: Different formulations of correlation: (a) traditional correlation compares activity at every time instant. In this case, the sequence at the top is perfectly correlated with the one just below it, but the correlation is weaker with the sequence at the bottom. (b) In this case, the time-lagged association with the sequence at the top relative to the symbol $\mathrm{X}$ is the same for each of the other two sequences. In the first case, for a time-lag of zero and a window length of 4 , the subsequence $\mathrm{ABBB}$ is always associated with the occurrence of $X$. In the second case, for a time-lag of zero and a window length of 4 , the subsequence $\mathrm{ABCD}$ is always associated with the occurrence of $\mathrm{X}$. In both cases, a consistent subsequence is associated with $\mathrm{X}$ and the entropy of activity associated with $\mathrm{X}$ is consequently 0 .
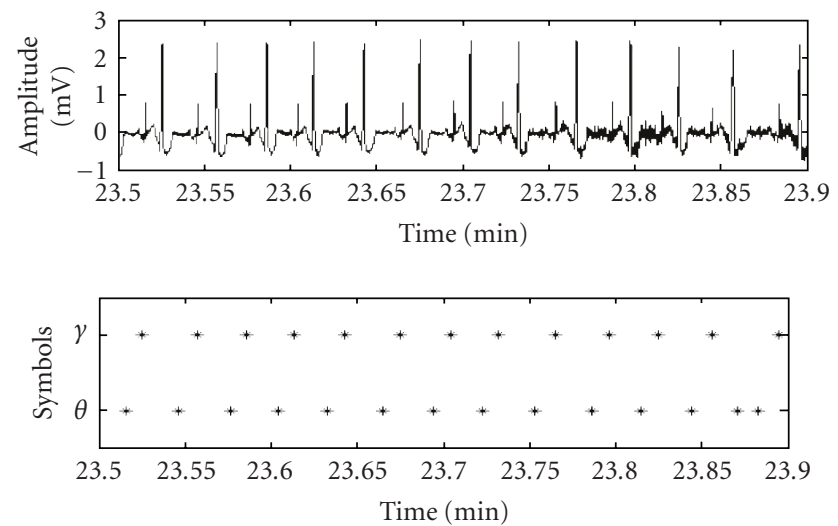

FIGURE 9: A patient with ventricular bigeminy.

the symbol string and facilitates viewing the signal over long time intervals. In each case, the repeating sequence in the symbolic signal corresponds to a well-known cardiac rhythm that can be recognized in the raw tracings. Figure 9 presents a signal showing a ventricular bigeminy pattern, while Figure 10 shows trigeminy. The associated symbolic streams provided for both figures show the repetitious activity in the reduced symbolic representations.

Figure 11 shows that our automated methods can be used to discover complex rhythms that are easy for clinicians to miss. In this case, approximate repeat detection identifies an 

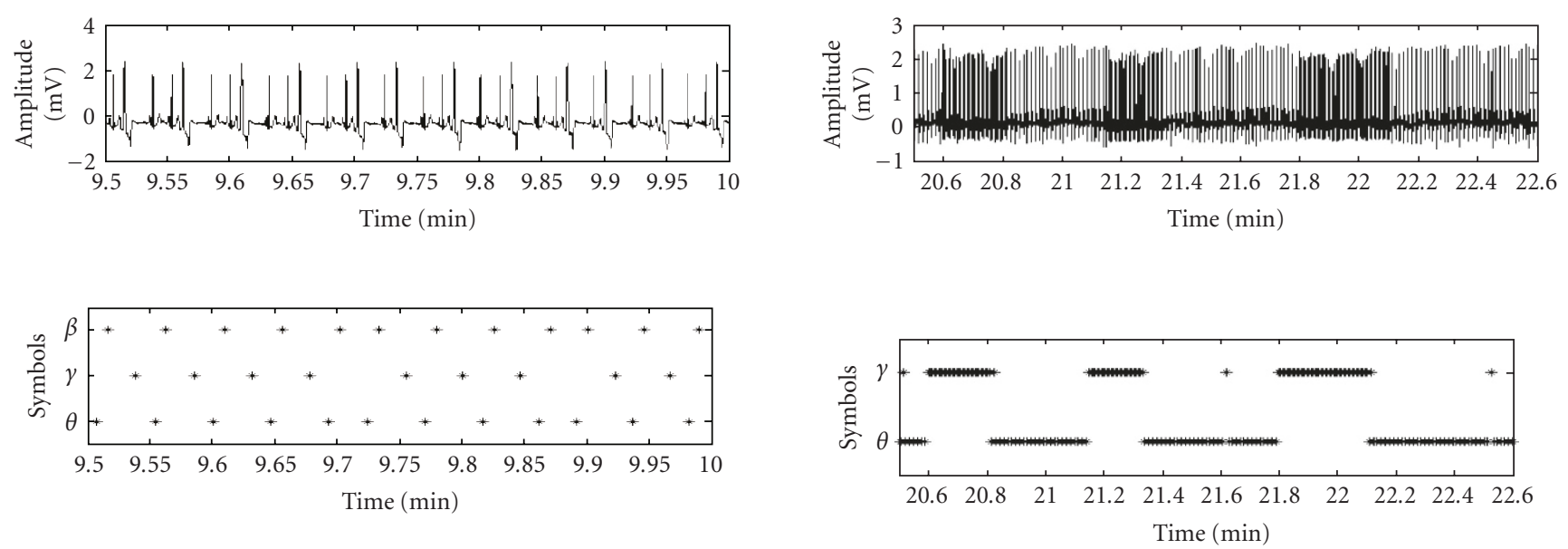

FIgURE 10: A patient with ventricular trigeminy.
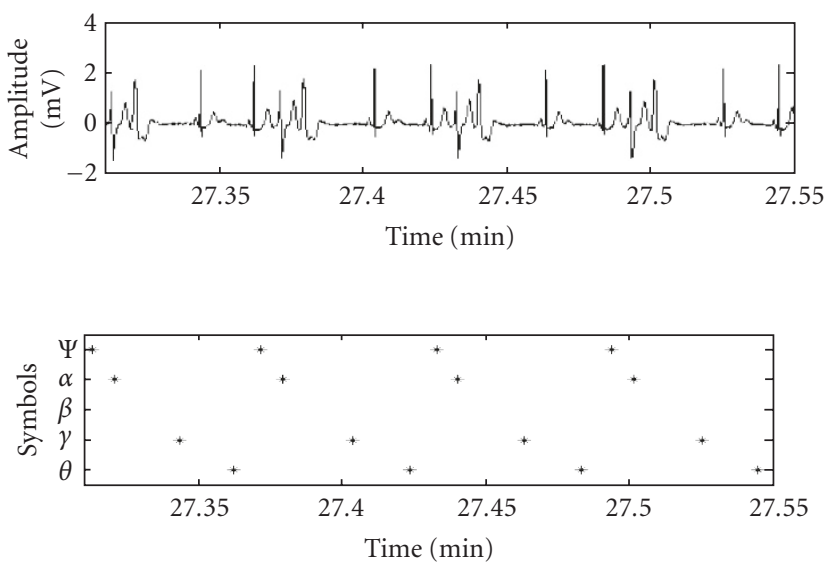

FIGURE 12: A patient with recurrent tachyarrhythmic episodes. These episodes appear in the raw tracing as dense regions, corresponding to an increased number of heart beats during these periods owing to faster heart rate.

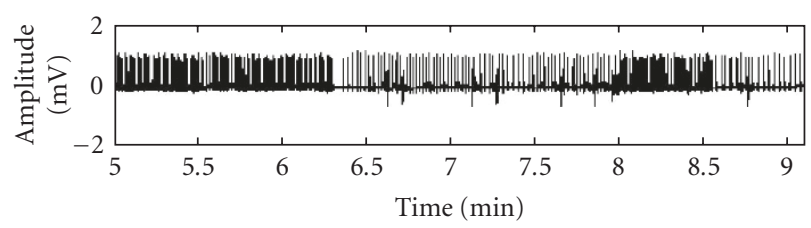

FIGURE 11: A rhythm of 4 units corresponding to an ectopic atrial rhythm.

intricate pattern which likely represents episodes of an ectopic atrial rhythm with aberrant ventricular conduction superimposed on an underlying sinus rhythm. This clinically significant rhythm was not marked by the clinicians who annotated the signal.

Figure 12 shows an example in which the detection of recurrent transient patterns in symbolic signals reveals many short, unsustained episodes of tachyarrhythmic activity. The tachyarrhytmic beats occur infrequently relative to normal beats, and consecutive runs of such activity are unlikely to have occurred merely at random.

Figure 13 presents the result of applying the techniques in Section 3.1.3 to discover high entropy segments corresponding to atrial fibrillation. The irregularity of activity leads to entropy increasing noticeably in windows of the symbolic stream, owing to the unstructured nature of the underlying disorder.
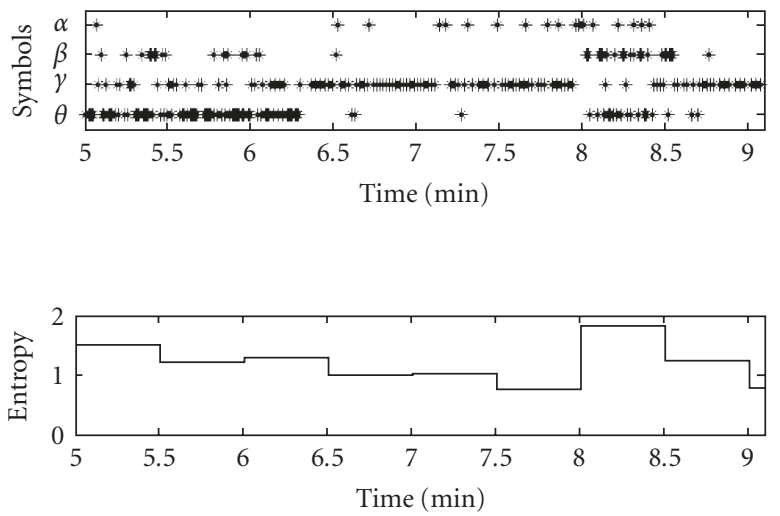

FIGURE 13: Raw ECG tracing, symbolic signal, and entropy taken over 30-second windows for a patient with atrial fibrillation. As in Figure 14, atrial fibrillation in the raw tracings corresponds to the dense regions.

\subsubsection{Analysis of multiple signals}

We tested our techniques designed to discover knowledge in multisignal datasets (Section 3.2) on the Physionet $\mathrm{MGH} / \mathrm{MF}$ Waveform database, comprising recordings across 3 ECG channels, ART, PAP, CVP, respiration and airway $\mathrm{CO} 2$, sampled at $360 \mathrm{~Hz}$ per channel with 12-bit quantization. 

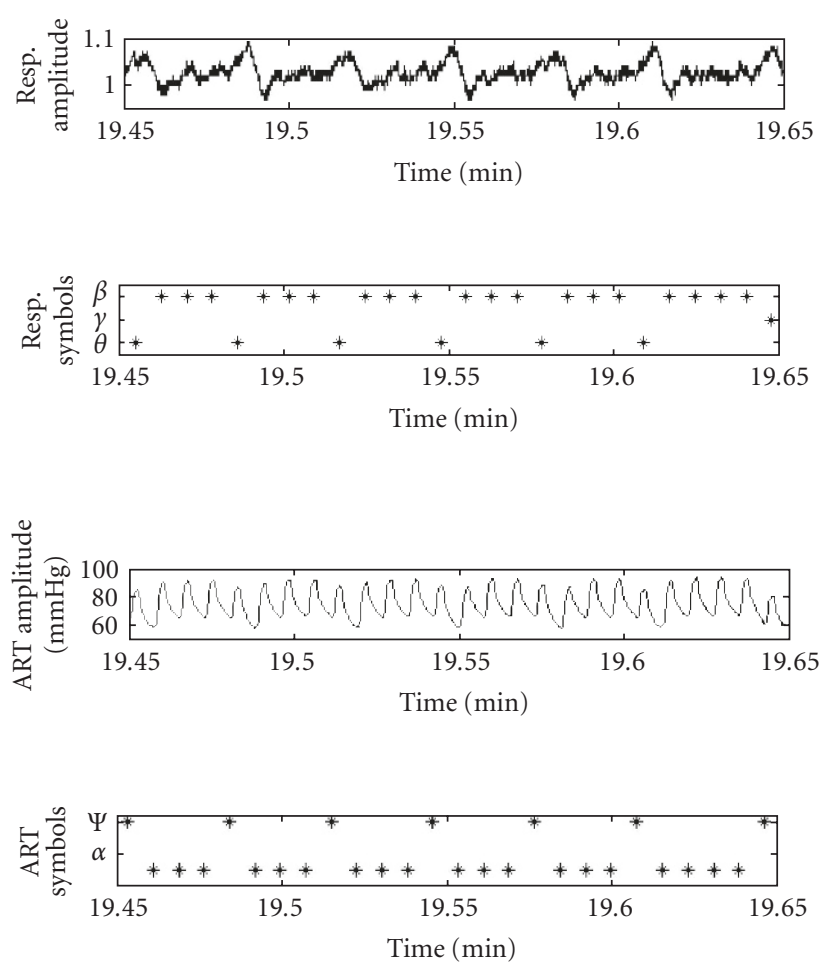

FIGURE 14: Respiration and arterial blood pressure signals for a patient with pulsus paradoxus.

Figures 14 and 15 demonstrate multisignal trend detection. In Figure 14, the search for correlated activity revealed a case of pulsus paradoxus, where inspiration is associated with a significant drop in arterial blood pressure. This is often associated with cardiac tamponade, severe COPD, pulmonary embolism, or right ventricular infarction. In Figure 15, episodes of faster heart rate can be seen to occur in conjunction with increased arterial blood pressure, a finding indicative of a hemodynamically significant rhythm. In both cases, associations between the symbolic representations allow for these phenomena to be easily detected.

\section{RELATED WORK}

The process of creating symbolic representations of physiologic signals has been extensively studied in the context of ECG. Holter monitors [20] use special purpose algorithms to distinguish between different clinical classes of electrophysiological activity. In contrast, we have developed generic techniques that do not assume any prior knowledge, and instead discriminate among activities based on nonparametric morphological differences. Our techniques are designed both to reproduce the results of these specialized techniques, and to obtain complementary information. In this sense, our work is closely related to [6], which presents a fairly comprehensive evaluation of various approaches to morphology-based clustering. The use of visual features such as time samples,
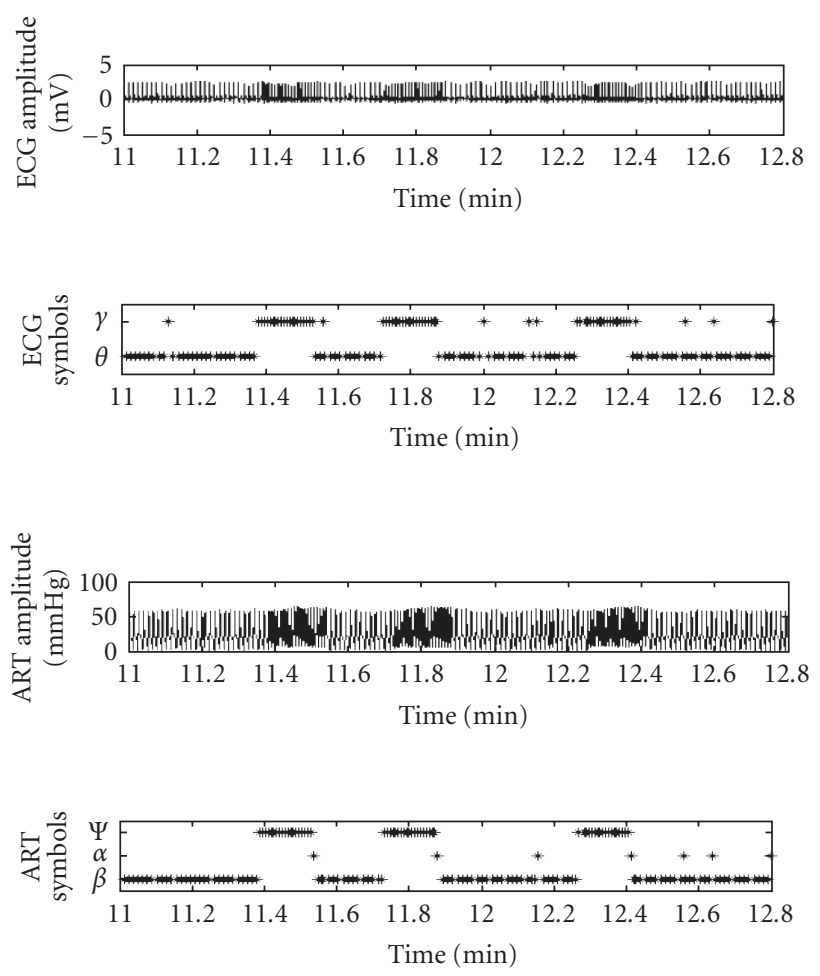

FIGURE 15: ECG and arterial blood pressure signals for a patient in whom fast heart rate leads to increased arterial blood pressure.

trace segmentation, polygonal approximation, and wavelet coefficients is discussed, and nonlinear alignment is also suggested to improve the quality of the clustering. We further examine morphology-based clustering through DTW from the perspective of separating clusters with a widely varying number of elements, for example long-term patient data where some groups of activity may have several orders of magnitude more members than others.

The goal addressed in [6] is to identify salient classes of activity. The resulting sequences are not further analyzed. In [21], stationary segments of EEG are clustered, and the original signal is replaced by the resulting sequences in a way resembling our approach. The focus of that work is to compress the original signal, and not on the analysis of the resulting sequence of symbols.

In [22] a method is presented for clustering QRS complexes using a basis function representation. The approach consists of: (1) segmenting ECG data streams according to the R-R interval; (2) expressing each beat as a linear combination of Hermite basis functions; and (3) clustering these beats using self-organizing neural networks [22]. Application to the MIT-BIH arrhythmia database results in 25 clusters with an overall misclassification error of only $1.5 \%$.

An important aspect of the method in [22] is that it performs electrocardiographic feature extraction using a basis set containing at most 6 Hermite functions. Each Hermite function contains a "width parameter" that enables one 
to effectively model QRS complexes with very different lengths. However, since the method relies on a relatively small set of basis functions, it may not fully capture subtle morphologic differences between beats. For example, in [22] the misclassification error for ventricular escape beats (E) is $37.7 \%$. The majority of these errors involve misclassifying ventricular beats as right bundle branch block (R). While beats belonging to the classes $\mathrm{E}$ and $\mathrm{R}$ both have long QRS durations, ventricular escape beats and right bundle branch block beats typically have significantly different morphologies [5].

Overall, our clustering approach performs marginally better on the MIT-BIH database. We found twenty-two clusters for the median patient (versus twenty-five in [22]) and had an overall misclassification error of $1.4 \%$ (versus $1.5 \%$ in [22]). Moreover, our method is based on comparing morphological characteristics of each beat and therefore does not make any assumption about the underlying form of each QRS complex. Consequently, our approach seems to be more sensitive to subtle variations in beat morphology. On the MIT-BIH database, for example, the misclassification error for beats within class $\mathrm{E}$ is only $6.7 \%$ - considerably lower than the $37.7 \%$ reported for the same database in [22].

We did not discover much prior work in the area of highlevel analysis of physiological symbolic sequences to uncover rhythms, patterns, and cross-signal interactions. A recent effort addressing this goal is described in [17]. In this case, symbolic strings are created corresponding to beat-by-beat changes in heart rate and blood pressure, and the evolution of the two signals is examined by means of joint symbolic dynamics, which measure simultaneous increases and decreases in both quantities.

\section{SUMMARY AND CONCLUSIONS}

In this paper, we presented and evaluated fully automated techniques for analyzing large amounts of cardiovascular data. Unlike traditional medical expert systems, which are aimed at detecting a prespecified set of events using a priori knowledge, we address the issue of discovering events with limited prior knowledge. Furthermore, since our techniques are intended to be applied to large data sets, for example, multiple days of continuous high-resolution ECG data, we place considerable emphasis on computational efficiency.

We focussed on transforming continuous waveform signals into symbolic strings derived directly from the data. Morphological features are used to partition beats into classes by maximizing the sequence-aligned separation of clusters, and the original signal is replaced by the corresponding sequence of symbols.

The symbolization process allows us to shift from the analysis of raw signals to the analysis of sequences of symbols. A discrete representation provides a layer of data reduction, making the search space for discovering interesting activity more manageable.

We described techniques that operate in this symbolic domain to discover cardiac activity of potential clinical im- portance. Our techniques automatically detect rhythms, transient patterns, high-entropy regions, and multisignals relationships.

We evaluated our techniques on files from 48 different patients drawn from the MIT-BIH arrhythmia public domain database of annotated ECG tracings. Our symbolization process placed beats in the same class as the cardiologist supplied annotations over $98.6 \%$ of the time, and for many of the differently classified beats the correct classification was arguable. In addition, our techniques allow for distinctions within clinical classes that could be relevant. We further tested our algorithm on a blind set of 40 patients from the $\mathrm{MGH} / \mathrm{MF}$ waveform database who were not used during the algorithm design process. In this case, our symbolization placed beats in the same class as the cardiologist-supplied annotations over $99.1 \%$ of the time.

The use of morphological features in conjunction with a DTW-based dissimilarity metric appeared to be sufficient for achieving a meaningful partitioning of the data in the case of ECG signals. Our modifications to the traditional DTW algorithm improve performance in the presence of additive noise and make the technique more sensitive to variations in length. The combined use of Max-Min clustering and a fuzzy preclustering phase allows the analysis of large amounts of data without excessive demands in terms of time or space.

Our higher level analysis techniques proved effective at identifying clinically relevant activity from symbolized ECG streams. Since the database did not label all occurrences of clinically relevant portions of the ECG, we were unable to evaluate the sensitivity of our analysis. We did have a cardiologist verify the specificity, and all of the detected sequences were indeed potentially clinically relevant. In one case, our techniques detected an ectopic atrial rhythm with aberrant ventricular conduction superimposed on an underlying sinus rhythm that had apparently gone undetected by the cardiologists compiling the data base.

We also demonstrated that our techniques aimed at identifying potentially relevant relationships across multiple symbolized streams, for example, streams representing ECG and respiratory data, could be used to find clinically significant activity.

Operating on a reduced symbolic representation of the original signals simplified the problem of discovering interesting activity. The search for many broad classes of clinical conditions could be posed in this symbolic domain, and a number of efficient techniques could be borrowed from computational biology and information theory.

Our techniques are intended to complement, not replace, existing methods. In scenarios where strong priors exist regarding the activity of interest, specialized detectors can be designed by factoring in known relationships between the signals and the underlying physiological activity may well out-perform our generic techniques. Furthermore, our techniques are not designed to provide definitive diagnoses, but rather to help professionals by making it easier for them to focus on the most relevant data. Correct interpretation of the data requires information, for example, clinical history, that is not currently incorporated in our methods. 
TABLE 4: Beats detected for each patient in the MIT-BIT arrhythmia database using symbolization. The error in this case is similar to that reported in Table 1, with an overall error once again of $1.37 \%$. The labels are identical to those in Table 2.

\begin{tabular}{|c|c|c|c|c|c|c|c|c|c|c|}
\hline Patient & $\mathrm{N}$ & $\mathrm{L}$ & $\mathrm{R}$ & A & $\mathrm{a}$ & $\mathrm{V}$ & $\mathrm{P}$ & $\mathrm{f}$ & $\mathrm{F}$ & $\mathrm{j}$ \\
\hline 100 & $2234 / 2234$ & - & - & $30 / 33$ & - & - & - & - & - & - \\
\hline 101 & $1852 / 1852$ & - & - & $3 / 3$ & - & - & - & - & - & - \\
\hline 102 & $14 / 99$ & - & - & - & - & $4 / 4$ & $2022 / 2023$ & $6 / 56$ & - & - \\
\hline 103 & $2076 / 2076$ & - & - & - & - & - & - & - & - & - \\
\hline 104 & $51 / 163$ & - & - & - & - & - & $1375 / 1375$ & $601 / 665$ & - & - \\
\hline 105 & $2530 / 2076$ & - & - & - & - & $39 / 40$ & - & - & - & - \\
\hline 106 & $1500 / 1500$ & - & - & - & - & $508 / 511$ & - & - & - & - \\
\hline 107 & - & - & - & - & - & $59 / 59$ & $2074 / 2075$ & - & - & - \\
\hline 108 & $1748 / 1748$ & - & - & $1 / 4$ & - & $15 / 16$ & - & - & - & - \\
\hline 109 & - & $2486 / 2486$ & - & - & - & $37 / 38$ & - & - & - & - \\
\hline 111 & $2117 / 2117$ & - & - & - & - & - & - & - & - & - \\
\hline 112 & $2533 / 2533$ & - & - & - & - & - & - & - & - & - \\
\hline 113 & $1782 / 1748$ & - & - & - & $5 / 5$ & - & - & - & - & - \\
\hline 114 & $1815 / 1815$ & - & - & $4 / 8$ & - & $42 / 44$ & - & - & $0 / 4$ & - \\
\hline 115 & $1946 / 1946$ & - & - & - & - & - & - & - & - & - \\
\hline 116 & $2281 / 2281$ & - & - & - & - & $107 / 107$ & - & - & - & - \\
\hline 117 & $1528 / 1528$ & - & - & - & - & - & - & - & - & - \\
\hline 118 & - & - & $2147 / 2161$ & $82 / 96$ & - & $16 / 16$ & - & - & - & - \\
\hline 119 & $1540 / 1540$ & - & - & - & - & $443 / 443$ & - & - & - & - \\
\hline 121 & $1858 / 1858$ & - & - & - & - & - & - & - & - & - \\
\hline 122 & $2475 / 2475$ & - & - & - & - & - & - & - & - & - \\
\hline 123 & $1510 / 1510$ & - & - & - & - & - & - & - & - & - \\
\hline 124 & - & - & $1523 / 1526$ & - & - & $47 / 47$ & - & - & $3 / 5$ & $3 / 5$ \\
\hline 200 & $1737 / 1739$ & - & - & $1 / 29$ & - & $796 / 814$ & - & - & - & - \\
\hline 201 & $1605 / 1605$ & - & - & $15 / 24$ & $49 / 52$ & $183 / 183$ & - & - & - & $3 / 10$ \\
\hline 202 & $2043 / 2046$ & - & - & $18 / 35$ & $10 / 13$ & $18 / 19$ & - & - & - & - \\
\hline 203 & $2432 / 2442$ & - & - & - & - & $318 / 344$ & - & - & - & - \\
\hline 205 & $2564 / 2565$ & - & - & $1 / 3$ & - & $66 / 66$ & - & - & $9 / 9$ & - \\
\hline 207 & - & $1453 / 1470$ & $85 / 89$ & $114 / 116$ & - & $92 / 103$ & - & - & - & - \\
\hline 208 & $1507 / 1575$ & - & - & - & - & $949 / 977$ & - & - & $311 / 371$ & - \\
\hline 209 & $2603 / 2617$ & - & - & $317 / 383$ & - & - & - & - & - & - \\
\hline 210 & $2411 / 2416$ & - & - & - & $14 / 21$ & $154 / 172$ & - & - & $4 / 10$ & - \\
\hline 212 & $920 / 920$ & - & $1821 / 1821$ & - & - & - & - & - & - & - \\
\hline 213 & $2632 / 2635$ & - & - & $3 / 25$ & $2 / 3$ & $192 / 219$ & - & - & $39 / 362$ & - \\
\hline 214 & - & $1980 / 1993$ & - & - & - & $259 / 260$ & - & - & - & - \\
\hline 215 & $3190 / 3191$ & - & - & - & - & $156 / 159$ & - & - & - & - \\
\hline 217 & $229 / 242$ & - & - & - & - & $138 / 157$ & $1540 / 1544$ & $164 / 258$ & - & - \\
\hline 219 & $2077 / 2077$ & - & - & $0 / 7$ & - & $31 / 62$ & - & - & - & - \\
\hline 220 & $1942 / 1947$ & - & - & $91 / 93$ & - & - & - & - & - & - \\
\hline 221 & $2028 / 2028$ & - & - & - & - & $381 / 382$ & - & - & - & - \\
\hline 222 & $1939 / 1977$ & - & - & $121 / 187$ & - & - & - & - & - & $125 / 215$ \\
\hline 223 & $2021 / 2025$ & - & - & $19 / 72$ & - & $457 / 470$ & - & - & $0 / 14$ & - \\
\hline 228 & $1685 / 1687$ & - & - & $0 / 3$ & - & $366 / 371$ & - & - & - & - \\
\hline 230 & $2249 / 2249$ & - & - & - & - & - & - & - & - & - \\
\hline 231 & $312 / 312$ & - & $1246 / 1247$ & - & - & - & - & - & - & - \\
\hline 232 & - & - & $435 / 437$ & $1407 / 1423$ & - & - & - & - & - & - \\
\hline 233 & $2219 / 2220$ & - & - & $0 / 7$ & - & $806 / 817$ & - & - & $1 / 11$ & - \\
\hline 234 & $2695 / 2696$ & - & - & - & - & $3 / 3$ & - & - & - & - \\
\hline Tot. Bts. & $76430 / 76802$ & $5919 / 5949$ & 7257/7281 & $2227 / 2551$ & $80 / 94$ & $6682 / 6903$ & $7011 / 7017$ & $771 / 979$ & $367 / 786$ & $131 / 230$ \\
\hline Tot. Clu. & $41 / 41$ & $3 / 3$ & $6 / 6$ & $16 / 19$ & $5 / 5$ & $29 / 29$ & $4 / 4$ & $3 / 3$ & $6 / 8$ & $3 / 3$ \\
\hline
\end{tabular}

\section{ACKNOWLEDGMENTS}

We would like to thank Manolis Kellis and Piotr Indyk for their insightful suggestions regarding the clustering process. We would also like to thank the anonymous reviewers and the editor whose many constructive suggestions led to considerable improvement in the manuscript. This work was supported by the Center for Integration of Medicine and Innovative Technology (CIMIT), the MIT Project Oxygen Partnership, the Burrough's Wellcome Fund and the Harvard-MIT Health Sciences and Technology (HST) Division. 


\section{REFERENCES}

[1] D. Kopec, M. H. Kabir, D. Reinharth, O. Rothschild, and J. A. Castiglione, "Human errors in medical practice: systematic classification and reduction with automated information systems," Journal of Medical Systems, vol. 27, no. 4, pp. 297-313, 2003.

[2] G. D. Martich, C. S. Waldmann, and M. Imhoff, "Clinical informatics in critical care," Journal of Intensive Care Medicine, vol. 19, no. 3, pp. 154-163, 2004.

[3] Z. Syed and J. Guttag, "Prototypical biological signals," in Proceedings of IEEE International Conference on Acoustics, Speech, and Signal Processing (ICASSP '07), Honolulu, Hawaii, U.S.A., April 2007.

[4] C. S. Daw, C. E. A. Finney, and E. R. Tracy, "A review of symbolic analysis of experimental data," Review of Scientific Instruments, vol. 74, no. 2, pp. 915-930, 2003.

[5] E. Braunwald, D. Zipes, and P. Libby, Heart Disease: A Textbook of Cardiovascular Medicine, WB Saunders, Philadelphia, $\mathrm{Pa}$, USA, 2001.

[6] D. Cuesta-Frau, J. C. Pérez-Cortés, and G. Andreu-García, "Clustering of electrocardiograph signals in computeraided Holter analysis," Computer Methods and Programs in Biomedicine, vol. 72, no. 3, pp. 179-196, 2003.

[7] C. S. Myers and L. R. Rabiner, "A comparative study of several dynamic time-warping algorithms for connected-word recognition," The Bell System Technical Journal, vol. 60, no. 7, pp. 1389-1409, 1981.

[8] D. L. Donoho, “De-noising by soft-thresholding," IEEE Transactions on Information Theory, vol. 41, no. 3, pp. 613-627, 1995.

[9] G. Chen, Q. Wei, and H. Zhang, "Discovering similar timeseries patterns with fuzzy clustering and DTW methods," in Proceedings of Joint 9th IFSA World Congress and 20th NAFIPS International Conference (NAFIPS '01), vol. 4, pp. 2160-2164, Vancouver, BC, Canada, July 2001.

[10] E. J. Keogh and M. J. Pazzani, "Scaling up dynamic time warping for data mining applications," in Proceeding of the 6th ACM SIGKDD International Conference on Knowledge Discovery and Data Mining (KDD '00), pp. 285-289, Boston, Mass, USA, August 2000.

[11] T. F. Gonzalez, "Clustering to minimize the maximum intercluster distance," Theoretical Computer Science, vol. 38, no. 23, pp. 293-306, 1985.

[12] J. Fraden and M. R. Neuman, "QRS wave detection," Medical and Biological Engineering and Computing, vol. 18, no. 2, pp. 125-132, 1980.

[13] R. Hamming, "Error-detecting and error-checking codes," The Bell System Technical Journal, vol. 29, no. 2, pp. 147-160, 1950.

[14] G. M. Landau, J. P. Schmidt, and D. Sokol, "An algorithm for approximate tandem repeats," Journal of Computational Biology, vol. 8, no. 1, pp. 1-18, 2001.

[15] S. F. Altschul, W. Gish, W. Miller, E. W. Myers, and D. J. Lipman, "Basic local alignment search tool," Journal of Molecular Biology, vol. 215, no. 3, pp. 403-410, 1990.

[16] D. Jennings, T. Amabile, and L. Ross, "Informal covariation assessments: data-based versus theory-based judgements," in Judgement Under Uncertainty: Heuristics and Biases, pp. 211230, Cambridge University Press, Cambridge, UK, 1982.

[17] M. Baumert, V. Baier, S. Truebner, A. Schirdewan, and A. Voss, "Short- and long-term joint symbolic dynamics of heart rate and blood pressure in dilated cardiomyopathy," IEEE Transac- tions on Biomedical Engineering, vol. 52, no. 12, pp. 2112-2115, 2005.

[18] N. Abramson, Information Theory and Coding, McGraw Hill, New York, NY, USA, 1963.

[19] I. Kojadinovic, "Relevance measures for subset variable selection in regression problems based on $k$-additive mutual information," Computational Statistics \& Data Analysis, vol. 49, no. 4, pp. 1205-1227, 2005.

[20] N. J. Holter, "New method for heart studies," Science, vol. 134, no. 3486, pp. 1214-1220, 1961.

[21] R. Agarwal, J. Gotman, D. Flanagan, and B. Rosenblatt, "Automatic EEG analysis during long-term monitoring in the ICU," Electroencephalography and Clinical Neurophysiology, vol. 107, no. 1, pp. 44-58, 1998.

[22] M. Lagerholm, C. Peterson, G. Braccini, L. Edenbrandt, and L. Sörnmo, "Clustering ECG complexes using hermite functions and self-organizing maps," IEEE Transactions on Biomedical Engineering, vol. 47, no. 7, pp. 838-848, 2000.

Zeeshan Syed received the S.B. and M.Eng. degrees in electrical engineering and computer science from the Massachusetts Institute of Technology (MIT), Cambridge, MA, in 2003, and is currently working towards a Ph.D. in the Harvard-MIT Health Sciences and Technology (HST) Division between the Department of Electrical Engineering and Computer Science, MIT, and Harvard Medical School. He has conducted

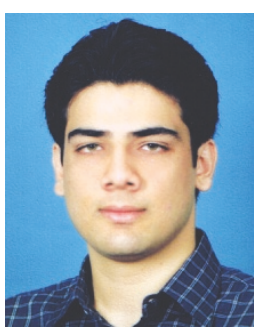
research at the Computer Science and Artificial Intelligence Laboratory (formerly Laboratory for Computer Science) at MIT, since 2001, working on projects related to the analysis and modeling of physiological signals, tools for the structured discovery of diagnostic markers, prototypical representations of biological activity, and the efficient detection of multimodal patterns in long-term data. His research interests include biomedical signal processing, machine learning, algorithms and computational biology. He is a Member of Sigma Xi, Tau Beta Pi, and Eta Kappa Nu. He is also a Recipient of the William A. Martin, Morris J. Levin Masterworks, and the Global Technovators awards at MIT.

John Guttag is the Dugald C. Jackson Professor of electrical engineering and computer science and a Member of the Computer Science and Artificial Intelligence Laboratory at the Massachusetts Institute of Technology (MIT), Cambridge, MA. Professor Guttag received A.B. and S.M. degrees from Brown University and his doctorate from the University of Toronto. He was on the faculty of the University of Southern

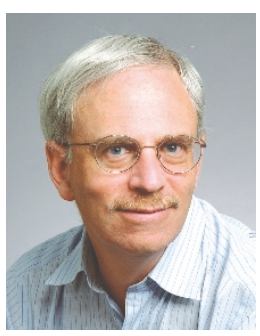
California from 1975-1978, and joined the MIT faculty in 1979. From 1993-1998, he served as Associate Department Head from Computer Science of MIT's Electrical Engineering and Computer Science Department, and served as Head of that department from 1999-2004. His current research interests include the application of wireless networking in healthcare, the development of techniques for the early detection of and interventions to control epileptic seizures, and techniques for analyzing large amounts of physiological data to discover information that can be used to assist in improving the management of chronic illness. 
Collin Stultz received his A.B. in mathematics and philosophy from Harvard College, magna cum laude, in 1988 and a Ph.D. in biophysics from the Graduate School of Arts and Sciences at Harvard University in 1997. That same year, he also earned an M.D., magna cum laude, from Harvard Medical School. He is a board certified cardiologist who trained at the Brigham and Women's Hospital in Boston. He is on the

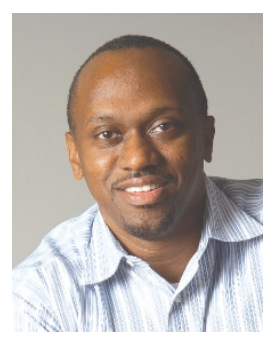
faculty in the Division of Health Sciences and Technology (HST) and MIT's Department of Electrical Engineering and Computer Science. He is a Member of the American Society for Biochemistry and Molecular Biology, the Federation of American Societies for Experimental Biology, the American Heart Association, and the American College of Cardiology. His research interests include the analysis of complex biological phenomena, including the structural dynamics of proteins and the interpretation of biological signals. 